\title{
Hospital-Associated Multicenter Outbreak of Emerging Fungus Candida auris, Colombia, 2016
}

\author{
Paige A. Armstrong, ${ }^{1}$ Sandra M. Rivera, ${ }^{1}$ Patricia Escandon, Diego H. Caceres, \\ Nancy Chow, Matthew J. Stuckey, Jorge Díaz, Adriana Gomez, Norida Vélez, Andres Espinosa-Bode, \\ Soraya Salcedo, Adriana Marin, Indira Berrio, Carmen Varón, Angel Guzman, Jairo E. Pérez-Franco, \\ Julian D. Escobar, Nohora Villalobos, Juan M. Correa, Anastasia P. Litvintseva, \\ Shawn R. Lockhart, Ryan Fagan, Tom M. Chiller, Brendan Jackson, ${ }^{2}$ Oscar Pacheco ${ }^{2}$
}

\section{Medscape ACTIVITY}

In support of improving patient care, this activity has been planned and implemented by Medscape, LLC and Emerging Infectious Diseases. Medscape, LLC is jointly accredited by the Accreditation Council for Continuing Medical Education (ACCME), the Accreditation Council for Pharmacy Education (ACPE), and the American Nurses Credentialing Center (ANCC), to provide continuing education for the healthcare team.

Medscape, LLC designates this Journal-based CME activity for a maximum of 1.00 AMA PRA Category 1 Credit(s) ${ }^{\mathrm{TM}}$. Physicians should claim only the credit commensurate with the extent of their participation in the activity.

Successful completion of this CME activity, which includes participation in the evaluation component, enables the participant to earn up to $1.0 \mathrm{MOC}$ points in the American Board of Internal Medicine's (ABIM) Maintenance of Certification (MOC) program. Participants will earn MOC points equivalent to the amount of CME credits claimed for the activity. It is the CME activity provider's responsibility to submit participant completion information to ACCME for the purpose of granting ABIM MOC credit.

All other clinicians completing this activity will be issued a certificate of participation. To participate in this journal CME activity: (1) review the learning objectives and author disclosures; (2) study the education content; (3) take the post-test with a $75 \%$ minimum passing score and complete the evaluation at http://www.medscape.org/journal/eid; and (4) view/print certificate. For CME questions, see page 1443.

Release date: June 13, 2019; Expiration date: June 13, 2020

Learning Objectives

Upon completion of this activity, participants will be able to:

- Assess the clinical characteristics and outcomes of a hospital-associated Candida auris outbreak in Colombia in 2015 to 2016 , based on a clinical case series

- Determine the likely mode of transmission and associated findings in a hospital-associated C. auris outbreak in Colombia in 2015 to 2016 , based on a clinical case series

- Evaluate treatment and other clinical and public health implications of a hospital-associated C. auris outbreak in Colombia in 2015 to 2016 , based on a clinical case series

CME Editor

Deborah Wenger, MBA, Copyeditor, Emerging Infectious Diseases. Disclosure: Deborah Wenger, MBA, has disclosed no relevant financial relationships.

CME Author

Laurie Barclay, MD, freelance writer and reviewer, Medscape, LLC. Disclosure: Laurie Barclay, MD, has disclosed no relevant financial relationships.

Authors

Disclosures: Paige A. Armstrong, MD, MHS; Sandra Rivera, BN, PGrad Dip; Patricia Escandon, MSc; Diego H. Caceres, MSc,; Nancy A. Chow, PhD; Matthew J. Stuckey, PhD, MPH; Jorge Luis Díaz, MD; Adriana Leonor Gomez, MS; Norida Natally Vélez, MSc; Andres Espinosa-Bode, MD; Soraya Salcedo, MD; Adriana Marin, MSc; Carmen Varón, BN, RN; Angel Alfonso Guzman, MD; Jairo E. Pérez-Franco, MD; Nohora Constanza Villalobos, MS; Juan Manuel Correa, MD, MSc; Anastasia P. Litvintseva, PhD; Shawn R. Lockhart, PhD; Ryan Fagan, MD, MPH\&TM; Tom Chiller, MD, MPH\&TM; Brendan R. Jackson, MD, MPH; and Oscar Pacheco, MD, have disclosed no relevant financial relationships. Indira Berrio, MD, has disclosed the following relevant financial relationships: served as an advisor or consultant for MSD, Pfizer; served as a speaker or a member of a speakers bureau for Gilead, MSD, Pfizer. Julian David Escobar, MSc, has disclosed the following relevant financial relationships: employed by a commercial interest, MSD Colombia.

Author affiliations: Centers for Disease Control and Prevention, Atlanta, Georgia, USA (P.A. Armstrong, D.H. Caceres, N. Chow, M.J. Stuckey, A. Espinosa-Bode, A.P. Litvintseva, S.R. Lockhart, R. Fagan, T.M. Chiller, B. Jackson); Instituto Nacional de Salud, Bogotá, Colombia (S.M. Rivera, P. Escandon, J. Díaz, A. Gomez, N. Vélez, O. Pacheco); Clínica General del Norte, Barranquilla, Colombia (S. Salcedo, A. Marin); Clínica El Rosario, Medellín, Colombia (I. Berrio); Corporación para Investigaciones Biológicas (CIB), Medellín (I. Berrio); Hospital General de Medellín,
Medellín (I. Berrio); Hospital Doña Pilar, Cartagena, Colombia

(C. Varón, A. Guzman); Hospital Militar Central, Bogotá

(J.E. Pérez-Franco, J.D. Escobar); Clínica los Nogales, Bogotá

(N. Villalobos, J.M. Correa)

DOI: https://doi.org/10.3201/eid2507.180491

${ }^{1}$ These authors contributed equally to this article.

${ }^{2}$ These authors contributed equally to this article. 
Candida auris is an emerging multidrug-resistant fungus that causes hospital-associated outbreaks of invasive infections with high death rates. During 2015-2016, health authorities in Colombia detected an outbreak of $C$. auris. We conducted an investigation to characterize the epidemiology, transmission mechanisms, and reservoirs of this organism. We investigated 4 hospitals with confirmed cases of $C$. auris candidemia in 3 cities in Colombia. We abstracted medical records and collected swabs from contemporaneously hospitalized patients to assess for skin colonization. We identified 40 cases; median patient age was 23 years (IQR 4 months-56 years). Twelve $(30 \%)$ patients were $<1$ year of age, and 24 $(60 \%)$ were male. The 30 -day mortality was $43 \%$. Cases clustered in time and location; axilla and groin were the most commonly colonized sites. Temporal and spatial clustering of cases and skin colonization suggest person-to-person transmission of $C$. auris. These cases highlight the importance of adherence to infection control recommendations.

C andida auris is an emerging multidrug-resistant fungus that has been implicated in recent hospital-associated outbreaks of invasive infections with high death rates (1). C. auris from an external ear isolate in Japan was described in 2009; the fungus has since been identified as the cause of outbreaks in other countries, with rapid spread globally (25). Of particular concern, C. auris isolates have demonstrated resistance to multiple classes of antifungal drugs $(2,6)$. In addition, whereas Candida is often considered a commensal organism that most commonly colonizes the gastrointestinal tract, the potential for person-to-person spread of $C$. auris has raised concern for widespread outbreaks $(7,8)$.

Accurate identification of $C$. auris is challenging because phenotypic methods do not correctly identify this species. It is most commonly misidentified as $C$. haemulonii, especially when using the VITEK 2 system (bioMérieux, https://www.biomerieux-diagnostics.com), but is also misidentified as C. famata, C. sake, Rhodotorula glutinis, and other Candida species (9). Specialized techniques, such as matrix-assisted laser desorption/ionization-time of flight (MALDI-TOF) mass spectrometry and DNA sequencing, are needed for reliable identification.

During January 2015-September 2016, the Colombian Instituto Nacional de Salud (INS) and the Secretarias de Salud (Ministries of Health) of Barranquilla, Bogotá, and Cartagena, Colombia, identified an increasing number of reported bloodstream infections with $C$. haemulonii, an otherwise rare yeast isolated in only a few invasive human infections (10). In May 2016, a total of 27 isolates initially identified as $C$. haemulonii by phenotypic identification methods were sent to the US Centers for Disease Control and Prevention (CDC), where 24 were confirmed to be $C$. auris by MALDI-TOF mass spectrometry (Microflex; Bruker Daltonics, https://www.bruker.com) and DNA sequencing. Through case findings and review of national surveillance data, an additional 16 cases of $C$. haemulonii reported to INS were identified as $C$. auris by MALDI-TOF mass spectrometry and confirmed at $\mathrm{CDC}$ with sequencing. The cases were reported from 4 large referral hospitals in Colombia. Two of these hospitals were located along the northern coast: 1 in Barranquilla, which serves both pediatric and adult patients, and the other a specialized pediatric facility in Cartagena. The other 2 hospitals were located in Bogotá and serve adult patients. No transfers took place between the involved facilities, and all follow national guidelines for infection control practices (11). These findings prompted an investigation to further characterize the epidemiology, transmission mechanisms, and environmental reservoirs of this organism to guide prevention measures.

\section{Methods}

In September 2016, a team consisting of clinicians, epidemiologists, and microbiologists from INS, CDC, and the Ministries of Health of Barranquilla, Bogotá, and Cartagena conducted an outbreak investigation in 1 acute-care hospital serving adult and pediatric patients in Barranquilla, 2 adult hospitals in Bogotá, and 1 pediatric hospital in Cartagena. These facilities all had confirmed cases of $C$. auris candidemia after January 2015.

\section{Descriptive Epidemiology}

We defined a case as $C$. auris confirmed by MALDI-TOF mass spectrometry isolated from a patient's blood during January 2015-September 2016. We abstracted medical records using a standardized case report form to collect data on concurrent conditions, location and duration of hospital stay, administration of antimicrobial drugs, exposure to invasive procedures, use of indwelling catheters, and outcome. We also interviewed infection control personnel, healthcare workers, and key informants at each site to trace exposures and locations of patients throughout their hospital stays.

\section{Sampling and Laboratory Analysis}

To better understand the role of colonization and transmission, we obtained samples from the 7 patients who were hospitalized at the time of the investigation with $C$. auris isolated or suspected in a specimen. We used premoistened swabs (Fisherfinest Transport Swabs, https://www. fishersci.com) to sample the following 10 body sites: bilateral nares, ears, axillae, and groin; oral cavity; and rectum. These locations were determined to be high-yield sites for colonization, and sampling was intended to provide initial insight into the utility of each. We collected fecal and urine specimens when possible.

Specimens were processed at the INS Microbiology Laboratory using protocols developed by CDC's Mycotic Diseases Branch (National Center for Emerging and Zoonotic Infectious Diseases, Division of Foodborne, Waterborne, 
and Environmental Diseases). In brief, the swabs were cultured in Sabouraud dextrose enrichment broth with high salt content $(10 \% \mathrm{wt} / \mathrm{vol} \mathrm{NaCl})$ at elevated temperature $\left(40^{\circ} \mathrm{C}\right)$, shaken $(250 \mathrm{rpm})$ for up to 7 days, and plated onto Sabouraud dextrose agar (12). Yeast isolates recovered from the selective agar were initially screened using CHROMagar Candida (Becton Dickinson, http://www.bd.com); white and pink colonies were typed using the Microflex database version MBT 6903 MSP Library (no. 1829023) using CDC MicrobeNet's supplemental MALDI database library.

Antifungal susceptibility testing was performed at CDC by broth microdilution using custom-made frozen panels; susceptibility testing for amphotericin B was performed using Etest (bioMérieux). We used the following breakpoints, based on published data: fluconazole $\geq 32 \mu \mathrm{g} /$ $\mathrm{mL}$, amphotericin $\mathrm{B} \geq 2 \mu \mathrm{g} / \mathrm{mL}$, caspofungin $\geq 4 \mu \mathrm{g} / \mathrm{mL}$, and anidulafungin $\geq 2 \mu \mathrm{g} / \mathrm{mL}$ (13).

\section{Statistical Analysis}

We calculated medians and ranges for continuous variables and frequencies and percentages for categorical variables. We used 30-day mortality as outcome of interest for analysis. To compare characteristics between patients who died and those who survived, we applied a $t$-test (equal or unequal variance as appropriate) for continuous variables and a $\chi^{2}$ test for categorical variables. For categorical variables having cell sizes $<5$, we used the Fisher exact test. Sample size did not allow for multivariate analysis. When comparing medians, we used Wilcoxon rank, as distributions were not normal. We performed all data analyses in SAS version 9.3 (https://www.sas.com) and considered a p value $<0.05$ significant.

CDC and INS determined that this was an emergency public health investigation. Therefore, it did not meet the criteria for research.

\section{Results}

We identified 40 cases of $C$. auris candidemia occurring during January 2015-September 2016. In 3 hospitals, cases

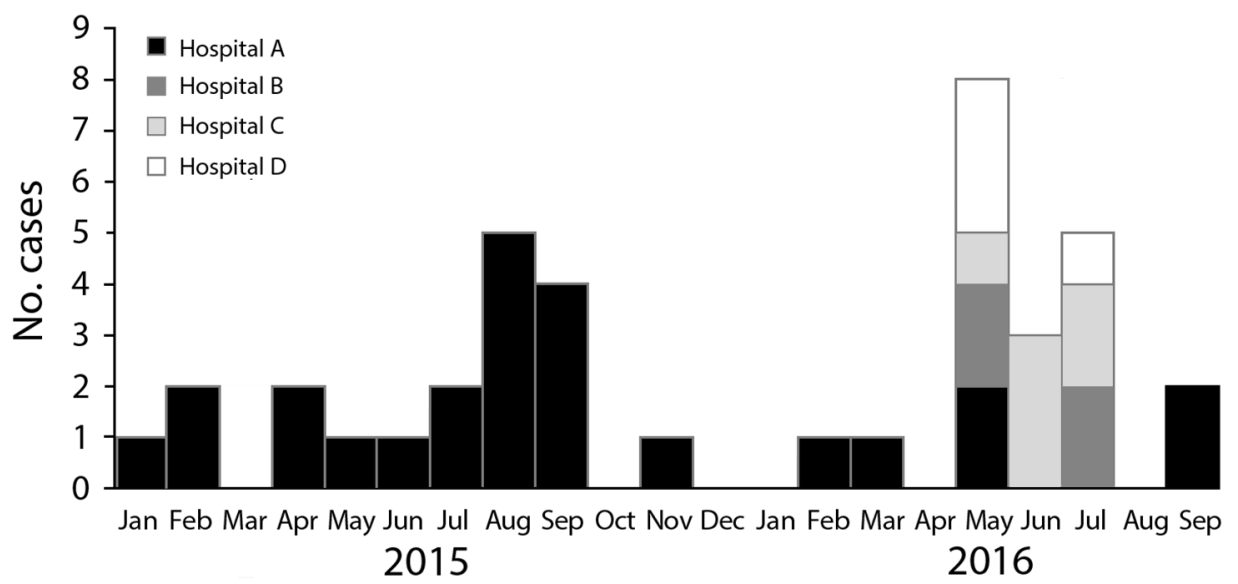

clustered in May-July 2016, whereas in the fourth hospital, cases occurred throughout the 21-month period (Figure 1). The median patient age was 23 years (interquartile range [IQR] 4 months-56 years); 12 patients (30\%) were $<1$ year of age. Most patients $(24 ; 60 \%)$ were male. Thirtyfive $(88 \%)$ patients had a documented concurrent condition before hospitalization; the most commonly reported conditions were hemodialysis for renal failure $(23 \%)$; diabetes (18\%); and immunocompromising conditions (16\%), including cancer and transplant.

More than half $(58 \%)$ of patients died while hospitalized; the overall 30 -day mortality was $43 \%$. The median length of admission was 46 days (IQR 34-69 days). For the 38 patients with known intensive care unit (ICU) admission, median length of ICU stay was 36 days (IQR 25-58 days). All case-patients were exposed to an invasive procedure. Central venous catheter (CVC) placement was present in all 40 patients $(100 \%)$; other common procedures were intubation $(35 ; 97 \%)$, surgical procedures $(28 ; 70 \%)$, and hemodialysis $(15 ; 38 \%)$ (Table 1$)$.

Median time from admission to having a blood culture positive for $C$. auris was 22 days (IQR 18-31 days). The median time from placement of CVC to positive blood culture was 12 days (IQR 5-21 days). The most common treatments received for $C$. auris candidemia were fluconazole $(16 ; 43 \%)$ and caspofungin $(13 ; 34 \%)$. Nineteen patients (45\%) received $>1$ antifungal drug; the median number of antimicrobial drugs administered to patients was 3.5 (range 1-6) (Table 1). The most frequent laboratory abnormalities were mild leukocytosis (median leukocyte count $12.7 \times 10^{9}$ cells/L) and anemia (median hemoglobin $9.8 \mathrm{~g} / \mathrm{dL}$ ).

The median age of the 17 patients who died within 30 days of positive culture was 36 years (IQR 6 months-65 years). Neither age group $(p=0.18)$, $\operatorname{sex}(p=0.9)$, nor preexisting concurrent condition $(p=0.30)$ were associated with 30-day mortality. Diabetes was associated with 30day mortality (odds ratio [OR] 12, 95\% CI 1.28-112.42; p $=0.03)$. The median length of hospital stay was longer for surviving case-patients (63 days, IQR 45-95 days) than

Figure. Epidemic curve for cases of Candida auris candidemia in Colombia, by hospital, January 2015-September 2016. 
Table 1. Characteristics of 40 patients with Candida auris bloodstream infection, Colombia, 2015-2016*

\begin{tabular}{|c|c|c|}
\hline Characteristic & Value & $\begin{array}{l}\text { Data } \\
\text { missing }\end{array}$ \\
\hline Median age (IQR) & 23 y $(4 \mathrm{mo}-56 \mathrm{y})$ & 0 \\
\hline Infant up to $1 \mathrm{y}$ & $12(30)$ & 0 \\
\hline Child $1-18$ y & $8(20)$ & 0 \\
\hline Adult $18-65$ y & $14(35)$ & 0 \\
\hline Adult $>65$ y & $6(15)$ & 0 \\
\hline \multicolumn{3}{|l|}{ Sex } \\
\hline $\mathrm{M}$ & $24(60)$ & 0 \\
\hline $\mathrm{F}$ & $16(40)$ & 0 \\
\hline Concurrent conditions & $35(88)$ & 0 \\
\hline Chronic renal disease & $9(23)$ & 0 \\
\hline Hemodialysis dependent & $9(23)$ & 0 \\
\hline Diabetes & $7(18)$ & 2 \\
\hline Immunosuppressive condition & $6(16)$ & 3 \\
\hline Cancer & $4(11)$ & 3 \\
\hline Solid tumor & $2(5)$ & 4 \\
\hline Hematologic malignancy & $2(5)$ & 4 \\
\hline Transplant & $2(5)$ & 4 \\
\hline Neuromuscular condition & $1(3)$ & 0 \\
\hline \multicolumn{3}{|l|}{ Outcome at $30 \mathrm{~d}$} \\
\hline Deceased & $17(43)$ & 0 \\
\hline Alive & $21(53)$ & 0 \\
\hline Hospitalized & $2(5)$ & 0 \\
\hline In-hospital deaths & $23(58)$ & 6 \\
\hline Admitted to hospital & $40(100)$ & 0 \\
\hline Median inpatient stay, d (IQR) & $46(34-69)$ & 3 \\
\hline Admitted to ICU & $38(100)$ & 2 \\
\hline Median ICU stay, d (IQR) & $36(25-58)$ & 10 \\
\hline Transferred from another facility & $16(40)$ & 0 \\
\hline \multicolumn{3}{|c|}{ Previously hospitalized in the $90 \mathrm{~d}$ before admission } \\
\hline Yes & $11(28)$ & 1 \\
\hline No & $23(59)$ & 1 \\
\hline Unknown & $5(13)$ & 1 \\
\hline \multicolumn{3}{|l|}{ Previous exposure to antifungal drugs } \\
\hline No & $22(67)$ & 7 \\
\hline Unknown & $11(33)$ & 7 \\
\hline \multicolumn{3}{|l|}{ Treatments and procedures } \\
\hline CVC & $40(100)$ & 0 \\
\hline Vasopressors & $31(82)$ & 2 \\
\hline Respiratory support & $36(100)$ & 4 \\
\hline Intubation & $35(97)$ & 4 \\
\hline Bilevel positive airway pressure & $1(3)$ & 4 \\
\hline Surgical procedure & $28(70)$ & 0 \\
\hline Total parenteral nutrition & $19(50)$ & 2 \\
\hline Corticosteroids & $18(47)$ & 2 \\
\hline Hemodialysis & $15(38)$ & 1 \\
\hline Bronchoscopy & 7 (19) & 4 \\
\hline Chemotherapy & $4(11)$ & 3 \\
\hline $\begin{array}{l}\text { Median time from admission to } C \text {. } \\
\text { auris positive culture, } d(I Q R)\end{array}$ & $22(18-31)$ & 0 \\
\hline $\begin{array}{l}\text { Median time from CVC to } C \text {. auris } \\
\text { positive culture, } d \text { (IQR) }\end{array}$ & $12(5-21)$ & 1 \\
\hline \multicolumn{2}{|c|}{ Treatment for $C$. auris bloodstream infection } & 0 \\
\hline Fluconazole & $16(42)$ & \\
\hline Caspofungin & $13(34)$ & \\
\hline Amphotericin B & $8(21)$ & \\
\hline Voriconazole & $1(3)$ & \\
\hline No antifungal & $2(5)$ & \\
\hline$>1$ antifungal & $19(48)$ & \\
\hline $\begin{array}{l}\text { No. antibacterial drugs } \\
\text { administered, median (range) }\end{array}$ & $3.5(1-6)$ & \\
\hline
\end{tabular}

for those who died (36 days, IQR 29-45 days; $\mathrm{p}<0.01$ ). The median length of ICU admission was 32 days (IQR 27-44 days) for case-patients who died and 51 days (IQR 14-76 days) for those who survived $(\mathrm{p}=0.3)$. No procedures were found to be associated with 30-day mortality. The median time from admission to positive blood culture was 22 days (IQR 21-37 days) for the patients who died and 21 days (IQR 12-28 days) in those who survived (p $=0.23$ ). The median time from positive blood culture to death in those who died was 7.5 days (IQR 6-17 days). We were unable to ascertain whether death was attributable to $C$. auris fungemia.

Among the 12 cases in infants, median age was 34 days (IQR $17-107$ days) and 9 (75\%) were male; $6(50 \%)$ died in the hospital, and $5(42 \%)$ died within 30 days of $C$. auris culture. Five $(50 \%)$ were preterm $(<37$ weeks' gestation), and $6(50 \%)$ had congenital heart disease (Table 2). Four $(80 \%)$ of 5 premature infants survived.

We performed antifungal susceptibility testing on 34 available clinical isolates from 34 unique case patients at 3 hospitals. Six (18\%) isolates were resistant to fluconazole, $10(29 \%)$ to amphotericin B, and none to the echinocandin class (anidulafungin, caspofungin). Ten $(50 \%)$ patients who died had been treated with fluconazole, 9 (43\%) received caspofungin, 1 received amphotericin $\mathrm{B}$, and 1 received voriconazole; 2 patients who died did not receive an antifungal drug. Of the 6 case-patients with isolates resistant to fluconazole, 4 (67\%) died by 30 days. Of the 10 case-patients with isolates resistant to amphotericin $\mathrm{B}, 4$ (40\%) died by 30 days. Resistance to fluconazole was not

\begin{tabular}{|c|c|c|}
\hline Characteristic & Value & $\begin{array}{c}\text { Data } \\
\text { missing }\end{array}$ \\
\hline Median age, d (IQR) & $34(17-107)$ & 0 \\
\hline \multicolumn{3}{|l|}{ Sex } \\
\hline M & $9(75)$ & 0 \\
\hline $\mathrm{F}$ & $3(25)$ & 0 \\
\hline 30-day mortality & $5(42)$ & 0 \\
\hline \multicolumn{3}{|l|}{ In-hospital mortality } \\
\hline Died & $6(50)$ & 0 \\
\hline Alive & $4(33)$ & 0 \\
\hline Still hospitalized, 2016 Sep 30 & $2(17)$ & 0 \\
\hline Concurrent conditions & $12(100)$ & 0 \\
\hline Prematurity & $5(50)$ & 2 \\
\hline $\begin{array}{l}\text { Median gestational age at birth, } \\
\text { wk (IQR) }\end{array}$ & $36(29-39)$ & 2 \\
\hline Congenital heart disease & $6(50)$ & 0 \\
\hline Surgical procedure & $9(75)$ & 0 \\
\hline \multicolumn{3}{|l|}{ Treatments and procedures } \\
\hline Central venous catheter & $12(100)$ & 0 \\
\hline Intubation & $11(100)$ & 1 \\
\hline Total parenteral nutrition & $11(92)$ & 0 \\
\hline Feeding tube & $5(71)$ & 5 \\
\hline Corticosteroids & $4(33)$ & 0 \\
\hline Hemodialysis & $1(8)$ & 0 \\
\hline
\end{tabular}


associated with outcome $(\mathrm{p}=0.21)$, nor was resistance to amphotericin B $(p=0.85)$ (Table 3). Fluconazole-resistant isolates were seen at all 3 hospitals and amphotericin B-resistant isolates at 2 hospitals, in Barranquilla and Cartagena (northern region of Colombia).

\section{Patient Sampling}

We collected skin and rectal swab specimens from 7 patients admitted at the time of the investigation: 3 patients with $C$. auris bloodstream infection, 2 with $C$. auris cultured from a body site other than blood, and 2 without a clinical $C$. auris culture (but who at the time had a clinical culture growing yeast suspected to be $C$. auris). Five patients yielded positive samples, 4 of whom had a clinical culture with $C$. auris. One patient with a bloodstream infection had no positive samples. Samples were collected 3 weeks to 3 months after positive clinical culture (Table 4). The case isolates showed a high degree of relatedness on whole-genome sequencing within hospitals and regional clustering, supporting in-hospital and person-to-person transmission (14).

We identified spatial and temporal clustering of cases in 1 operating room and multiple intensive care units at all 4 sites. Hospital A (Figure) experienced the largest number of cases over the longest period. Few case-patients shared the same room, and cases occurred throughout different floors and units. For the remaining hospitals, all cases occurred within a 3-month period. At hospital B (Figure), all 4 cases were exposed to the same operating room over a 3-month period. In hospitals $\mathrm{C}$ and $\mathrm{D}$, patients also overlapped in time and ICUs (Figure).

\section{Discussion}

Cases of C. auris bloodstream infection in Colombia were associated with nearly $60 \%$ all-cause, in-hospital deaths and a $43 \% 30$-day mortality, which is higher than that reported in the United States. Cases occurred primarily in patients in ICUs who had central venous catheters and other invasive devices, continuing to support the findings that these cases occur in patients with long stays involving multiple procedures (13). In contrast to the United States, where most cases have occurred in older adults, nearly one third of cases in Colombia were in infants, and the median patient age was 23 years (15). Although cases are occurring in vulnerable extremes of age populations, our findings suggest that no age group is unaffected and that any given hospital could be affected by an outbreak.

Clustering of patients in time and space, the findings of skin colonization, and the highly clonal nature of the

Table 3. Characteristics of patients who died at $30 \mathrm{~d}$ compared with those who survived Candida auris bloodstream infection, Colombia, 2015-2016*

\begin{tabular}{|c|c|c|c|c|c|}
\hline Characteristic & Total & Died & Survived & $\begin{array}{l}\text { Crude odds ratio } \\
(95 \% \mathrm{Cl})\end{array}$ & $\mathrm{p}$ value \\
\hline All & $40(100)$ & $17(43)$ & $23(58)$ & NA & NA \\
\hline Age, y & & & & & 0.18 \\
\hline$<1$ & $12(30)$ & $5(29)$ & $7(30)$ & $0.95(0.24-3.75)$ & 0.94 \\
\hline $1-17$ & $8(20)$ & $2(12)$ & $6(26)$ & $0.24(0.04-1.45)$ & 0.12 \\
\hline $18-65$ & $1435)$ & $5(29)$ & $9(39)$ & $0.65(0.17-2.47)$ & 0.53 \\
\hline$>65$ & $6(15)$ & $5(29)$ & $1(4)$ & $9.17(0.96-87.79)$ & 0.05 \\
\hline Sex & & & & $0.92(0.26-3.30)$ & 0.9 \\
\hline $\mathrm{M}$ & $24(60)$ & $10(59)$ & $14(61)$ & & \\
\hline $\mathrm{F}$ & $16(40)$ & $7(41)$ & $9(39)$ & & \\
\hline Concurrent conditions & $35(88)$ & $16(94)$ & $19(83)$ & $3.37(0.34-33.26)$ & 0.30 \\
\hline Chronic renal disease & $9(23)$ & $3(18)$ & $6(26)$ & $0.61(0.13-2.88)$ & 0.53 \\
\hline Hemodialysis dependent & $9(23)$ & $3(18)$ & $6(26)$ & $0.61(0.13-2.88)$ & 0.53 \\
\hline Diabetes & $7(18)$ & $6(35)$ & $1(4)$ & $12(1.28-112.42)$ & 0.03 \\
\hline Immunosuppressive condition & $6(16)$ & $4(24)$ & $2(9)$ & $3.23(0.52-20.20)$ & 0.21 \\
\hline Prematurity, $\mathrm{n}=12$ & $5(42)$ & $1(6)$ & $4(17)$ & $0.30(0.03-2.93)$ & 0.30 \\
\hline Median length of hospital stay, d (IQR) & $46(34-69)$ & $36(29-45)$ & $63(45-95)$ & NA & $<0.01$ \\
\hline Median length of ICU stay, d (IQR) & $36(25-58)$ & $32(27-44)$ & $51(14-76)$ & NA & 0.3 \\
\hline Central venous catheter & $40(100)$ & $17(100)$ & $23(100)$ & NA & NA \\
\hline Respiratory support & $36(90)$ & $17(100)$ & $19(83)$ & NA & 0.12 \\
\hline Vasopressors & $31(82)$ & $16(94)$ & $15(65)$ & $8.53(0.95-76.63)$ & 0.06 \\
\hline Surgical procedure & $28(70)$ & $13(76)$ & $15(65)$ & $1.73(0.43-7.11)$ & 0.45 \\
\hline Total parenteral nutrition & $19(50)$ & $7(41)$ & $12(52)$ & $0.64(0.18-2.28)$ & 0.49 \\
\hline Corticosteroids & $18(47)$ & $8(47)$ & $10(43)$ & $1.16(0.33-4.07)$ & 0.82 \\
\hline Hemodialysis & $15(38)$ & $8(47)$ & $7(30)$ & $2.03(0.55-7.47)$ & 0.29 \\
\hline Chemotherapy & $4(11)$ & $2(12)$ & $2(9)$ & $1.4(0.18-11.08)$ & 0.74 \\
\hline $\begin{array}{l}\text { Median time from admission to } C \text {. auris } \\
\text { positive culture, d (IQR) }\end{array}$ & $22(18-31)$ & $22(21-37)$ & $21(12-28)$ & NA & 0.23 \\
\hline Isolates resistant to fluconazole, $n=34$ & $6(18)$ & $4(24)$ & $2(9)$ & $3.23(0.52-20.2)$ & 0.21 \\
\hline Isolates resistant to amphotericin $\mathrm{B}, \mathrm{n}=34$ & $10(29)$ & $4(24)$ & $6(26)$ & $0.87(0.20-3.74)$ & 0.85 \\
\hline
\end{tabular}

†Fisher exact test, $\chi^{2}, t$-test, or Wilcoxon rank as appropriate. 
Table 4. Patient sampling in suspected or known cases of Candida auris fungemia, Colombia, $2016^{*}$

\begin{tabular}{lccc}
\hline Patient no. & $\begin{array}{c}\text { Primary specimen type } \\
\text { positive }\end{array}$ & $\begin{array}{c}\text { No. body sites sampled } \\
\text { for colonization }\end{array}$ & No. (\%) body sites positive for C. auris \\
\hline 1 & Blood & 11 & $1(9 \%):$ rectum \\
2 & Blood & 10 & 0 \\
3 & Blood & 11 & $7(64 \%)$ : ears, axilla, left nostril, rectum, fecal \\
material & $1(10 \%):$ left groin \\
4 & & 10 & $1(9 \%):$ right groin \\
5 & Urine & 11 & $2(20 \%):$ axillae \\
6 & Sputum & 10 & \\
\hline
\end{tabular}

isolates all strongly support person-to-person transmission occurring in the healthcare setting. Although Candida is often considered a commensal organism that most commonly colonizes the gastrointestinal tract, C. auris behaves more similarly to C. parapsilosis in its propensity to colonize the skin, which provides an opportunity for person-to-person spread. Outbreaks of C. parapsilosis have occurred in both adult and neonatal ICUs $(16,17)$. In our investigation, 2 NICUs were involved; in at least 1 NICU, healthcare workers shared time between patients, providing an opportunity for transmission. The propensity of $C$. auris to colonize the skin may also explain the strong association with intubation, catheters, and feeding tubes in infants. The ability of $C$. auris to form biofilms may further enhance its ability to migrate into the bloodstream when provided a conduit through the skin (18). In this investigation, central lines were in place for a mean of 12 days at the time of positive culture. The tendency for skin colonization, biofilm production, and ability to cause invasive infection further emphasizes the need for diligent central line care.

The high death rate associated with $C$. auris infections, coupled with potential for nosocomial transmission, including among high-risk neonates, underscores the importance of infection control to prevent its spread. Current recommendations focus on early notification, hand hygiene, disinfection with a US Environmental Protection Agency-registered hospital-grade disinfectant effective against Clostridium difficile spores, and use of standard and contact precautions (19).

The characteristics of patients with $C$. auris candidemia were similar, but not identical, to those reported for candidemia caused by other species. Patients with $C$. auris bloodstream infection shared exposures such as ICU stay, central venous catheters, and surgical procedures (7). Whereas immunosuppressive conditions, including solid organ tumors, hematologic malignancy, and transplants are known risk factors for candidemia, they were uncommon in patients with $C$. auris candidemia (18). The reason for this may be that whereas other Candida species invade when there is a shift in the physiology of the host, $C$. auris is not a commensal, but rather a new exposure. Thirty-day mortality rates for $C$. auris candidemia in
Colombia appear to be slightly higher (43\%) than those seen for candidemia from other species, although the small sample size in our investigation limits the comparison (20). Half the infants in our cohort died, although age was not significantly associated with death. Furthermore, we compared characteristics between case-patients who survived and those who died and found few to be associated with outcome. Preterm birth was more common in those who survived, and only diabetes was associated with 30-day mortality. The high death rate is likely multifactorial but is most clearly related to long hospital stays, multiple invasive procedures, and lack of clinician awareness of resistance profiles.

In September 2016, a national laboratory alert was released in Colombia to increase reporting and surveillance catchment, as well as to enhance awareness of $C$. auris and the other species with which it was most commonly confused (21). According to the national guidelines for surveillance of healthcare-associated infections, aerobic and anaerobic blood cultures should be performed on any patient with signs of bloodstream infection (11). However, the capacity to perform blood cultures may not be available in all facilities; thus, it is possible that cases may be missed. The alert coincided with the initiation of our investigation and thus did not contribute heavily to our case counts, but it did increase reporting nationally.

Directed treatment is key to successful outcomes in fungemia. Resistance is another noteworthy feature of $C$. auris. Among C. auris isolates collected from 4 world regions, nearly all $(93 \%)$ were resistant to fluconazole, and $41 \%$ were resistant to $\geq 2$ classes of antifungal drugs (13). In our investigation, fluconazole was the most common antifungal drug administered, and treatment failure may have played a role in death rates. Of the isolates in this investigation, only about one fifth were resistant to fluconazole, but nearly one third were resistant to amphotericin B. The low proportion resistant to fluconazole was surprising; medical practices surrounding administration likely play a role. Forty-three percent of patients received fluconazole around the time of blood culture yielding $C$. auris, and of these, $57 \%$ died. The mortality rate was even higher $(83 \%)$ among the patients with isolates resistant to fluconazole. Most $C$. auris isolates have been susceptible to 
echinocandin drugs, which remain the recommended firstline treatment for $C$. auris candidemia $(9,13,22)$. However, the often high cost of these drugs can be an obstacle to their use in resource-limited settings.

Because of the retrospective nature of this investigation, data on all variables were not available for each casepatient. Data on previous exposure to antifungal drugs and laboratory values were the most commonly missing. These limitations, along with the small sample size, may have prevented the detection of associations between risk factors and outcome.

The findings of our investigation highlight the necessity of adherence to infection control recommendations, especially aspects of careful central line care and maintenance, hand hygiene, proper disinfection of medical equipment, and use of standard and contact precautions (https://www.cdc.gov/ fungal/diseases/candidiasis/recommendations.html). C. auris remains an emerging pathogen with the potential for high levels of resistance to a limited body of antifungal drugs. Its propensity to colonize skin provides a means for person-to-person transmission and elevates the concern for healthcare-associated outbreaks. Further understanding of its comportment and attention to infection control and prevention recommendations are paramount to prevent further spread.

\section{Acknowledgments}

We acknowledge Claudia Parra and the Pontificia

Universidad Javeriana for diagnostic support during the course of this investigation; Martha Lucia Ospina and Maritza Gonzalez for support in the development this study; and Reina Turcios and Loren Cadena. We also acknowledge contributions from Maria Victoria Ovalle; the infection control teams of the Ministries of Health of Bogotá, Cartagena, and Barranquilla; and Cary Hilbert for their assistance in data collection and chart review. Paige Armstrong and Matthew Stuckey acknowledge the Epidemic Intelligence Service, as the investigation took place during their participation in the program. Diego Caceres acknowledges Oak Ridge Institute for Science and Education (ORISE).

This work was supported by the Centers for Disease Control and Prevention and the Instituto Nacional de Salud de Colombia.

\section{About the Author}

Dr. Armstrong is a medical epidemiologist in the Division of Vector-Borne Diseases, National Center for Emerging and Zoonotic Infectious Diseases, Centers for Disease Control and Prevention, Atlanta, Georgia, USA; her research focuses on characterizing epidemiologic trends in infectious diseases and early intervention. Ms. Rivera is a nurse epidemiologist with training in infection control who currently works at the National Institute of Health of Colombia and is responsible for national surveillance and infection control for healthcareassociated infections.

\section{References}

1. Calvo B, Melo ASA, Perozo-Mena A, Hernandez M, Francisco EC, Hagen F, et al. First report of Candida auris in America: clinical and microbiological aspects of 18 episodes of candidemia. J Infect. 2016;73:369-74. http://dx.doi.org/10.1016/j.jinf.2016.07.008

2. Morales-López SE, Parra-Giraldo CM, Ceballos-Garzón A, Martínez HP, Rodríguez GJ, Álvarez-Moreno CA, et al. Invasive infections with multidrug-resistant yeast Candida auris, Colombia. Emerg Infect Dis. 2017;23:162-4. http://dx.doi.org/ 10.3201/eid2301.161497

3. Chowdhary A, Sharma C, Duggal S, Agarwal K, Prakash A, Singh PK, et al. New clonal strain of Candida auris, Delhi, India. Emerg Infect Dis. 2013;19:1670-3. http://dx.doi.org/10.3201/ eid1910.130393

4. Magobo RE, Corcoran C, Seetharam S, Govender NP. Candida auris-associated candidemia, South Africa. Emerg Infect Dis. 2014;20:1250-1. http://dx.doi.org/10.3201/eid2007.131765

5. Ruiz Gaitán AC, Moret A, López Hontangas JL, Molina JM, Aleixandre López AI, Cabezas AH, et al. Nosocomial fungemia by Candida auris: first four reported cases in continental Europe. Rev Iberoam Micol. 2017;34:23-7. http://dx.doi.org/10.1016/j. riam.2016.11.002

6. Chowdhary A, Anil Kumar V, Sharma C, Prakash A, Agarwal K, Babu R, et al. Multidrug-resistant endemic clonal strain of Candida auris in India. Eur J Clin Microbiol Infect Dis. 2014;33:919-26. http://dx.doi.org/10.1007/s10096-013-2027-1

7. Kullberg BJ, Arendrup MC. Invasive candidiasis. N Engl J Med. 2015;373:1445-56. http://dx.doi.org/10.1056/NEJMra1315399

8. Cleveland AA, Farley MM, Harrison LH, Stein B, Hollick R, Lockhart SR, et al. Changes in incidence and antifungal drug resistance in candidemia: results from population-based laboratory surveillance in Atlanta and Baltimore, 2008-2011. Clin Infect Dis. 2012;55:1352-61. http://dx.doi.org/10.1093/cid/cis697

9. Centers for Disease Control and Prevention. Candida auris interim recommendations for healthcare facilities and laboratories. 2016 [cited 2017 Feb 3]. https://www.cdc.gov/fungal/diseases/ candidiasis/recommendations.html

10. Cendejas-Bueno E, Kolecka A, Alastruey-Izquierdo A, Theelen B, Groenewald M, Kostrzewa M, et al. Reclassification of the Candida haemulonii complex as Candida haemulonii (C. haemulonii group I), C. duobushaemulonii sp. nov. (C. haemulonii group II), and C. haemulonii var. vulnera var. nov.: three multiresistant human pathogenic yeasts. J Clin Microbiol. 2012;50:3641-51. http://dx.doi.org/10.1128/JCM.02248-12

11. Sandra MRV, Liliana IBG, Andrea PVR. Infecciones asociadas a dispositivos; protocolo de vigilancia en salud publica. Instituto Nacional de Salud Colombia. 2017 [cited 2018 Dec 10]. http://www.ins.gov.co/buscador-eventos/ZIKA\%20Lineamientos/ PRO\%20Infecciones\%20aso\%20dispositivos_pdf

12. Welsh RM, Bentz ML, Shams A, Houston H, Lyons A, Rose LJ, et al. Survival, persistence, and isolation of the emerging multidrug-resistant pathogenic yeast Candida auris on a plastic health care surface. J Clin Microbiol. 2017;55:2996-3005. http://dx.doi.org/10.1128/JCM.00921-17

13. Lockhart SR, Etienne KA, Vallabhaneni S, Farooqi J, Chowdhary A, Govender NP, et al. Simultaneous emergence of multidrugresistant Candida auris on 3 continents confirmed by wholegenome sequencing and epidemiological analyses. Clin Infect Dis. 2017;64:134-40. http://dx.doi.org/10.1093/cid/ciw691

14. Escandón P, Chow NA, Caceres DH, Gade L, Berkow EL, Armstrong P, et al. Molecular epidemiology of Candida auris in Colombia reveals a highly related, countrywide colonization with regional patterns in amphotericin B resistance. Clin Infect Dis. 2019;68:15-21.

15. Vallabhaneni S, Kallen A, Tsay S, Chow N, Welsh R, Kerins J, et al. Investigation of the first seven reported cases of Candida 
auris, a globally emerging invasive, multidrug-resistant fungusUnited States, May 2013-August 2016. Am J Transplant. 2017;17:296-9. http://dx.doi.org/10.1111/ajt.14121

16. Pinhati HMS, Casulari LA, Souza ACR, Siqueira RA, Damasceno CMG, Colombo AL. Outbreak of candidemia caused by fluconazole resistant Candida parapsilosis strains in an intensive care unit. BMC Infect Dis. 2016;16:433 http://dx.doi.org/10.1186/s12879-016-1767-9

17. Hernández-Castro R, Arroyo-Escalante S, Carrillo-Casas EM, Moncada-Barrón D, Alvarez-Verona E, Hernández-Delgado L, et al. Outbreak of Candida parapsilosis in a neonatal intensive care unit: a health care workers source. Eur J Pediatr. 2010;169:783-7. http://dx.doi.org/10.1007/s00431-009-1109-7

18. Lortholary O, Renaudat C, Sitbon K, Madec Y, Denoeud-Ndam L, Wolff M, et al.; French Mycosis Study Group. Worrisome trends in incidence and mortality of candidemia in intensive care units (Paris area, 2002-2010). Intensive Care Med. 2014;40:1303-12. http://dx.doi.org/10.1007/s00134-014-3408-3

19. Centers for Disease Control and Prevention. Recommendations for infection control for Candida auris; infection control recommendations for inpatient settings (acute care hospitals, longterm acute care hospitals, and nursing homes). 2017 [cited 2017
Oct 2]. https://www.cdc.gov/fungal/diseases/candidiasis/ c-auris-infection-control.html

20. Ortíz Ruiz G, Osorio J, Valderrama S, Álvarez D, Elías Díaz R, Calderón J, et al. Risk factors for candidemia in non-neutropenic critical patients in Colombia. Med Intensiva. 2016;40:139-44.

21. Instituto Nacional de Salud Colombia. Alerta por emergencia global de infecciones invasivas causadas por la levadura multirresistente, Candida auris. 2016 [cited 2018 Dec 10]. https://www.minsalud.gov.co/sites/rid/Lists/BibliotecaDigital/ RIDE/IA/INS/ins-alerta-colombia-candida-auris.pdf

22. Pappas PG, Kauffman CA, Andes DR, Clancy CJ, Marr KA, Ostrosky-Zeichner L, et al. Executive summary: clinical practice guideline for the management of candidiasis: 2016 update by the Infectious Diseases Society of America. Clin Infect Dis. 2016;62:409-17. http://dx.doi.org/10.1093/cid/civ1194

Address for correspondence: Paige A. Armstrong, Centers for Disease Control and Prevention, 1600 Clifton Road NE, Mailstop C-17, Atlanta, GA 30329-4027, USA; email: yzu9@cdc.gov; Sandra Milena Rivera Vargas, Instituto Nacional de Salud, Av calle 26 N 51-20, Bogotá, Colombia; email: srivera@ins.gov.co

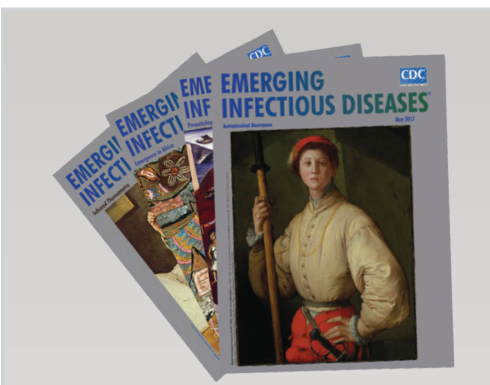

- Exposure Characteristics of Hantavirus Pulmonary Syndrome Patients, United States, 1993-2015

- Increased Neurotropic Threat from Burkholderia pseudomallei Strains with a B. mallei-like Variation in the bimA Motility Gene, Australia

- Population Genomics of Legionella longbeachae and Hidden Complexities of Infection Source Attribution

- Prevention of Chronic Hepatitis B after 3 Decades of Escalating Vaccination Policy, China

- Lack of Durable Cross-Neutralizing Antibodies against Zika Virus from Dengue Virus Infection

- Use of Blood Donor Screening Data to Estimate Zika Virus Incidence, Puerto Rico, April-August 2016

- Invasive Nontuberculous Mycobacterial Infections among Cardiothoracic Surgical Patients Exposed to Heater-Cooler Devices

- Anthrax Cases Associated with Animal-Hair Shaving Brushes

- Increasing Macrolide and Fluoroquinolone Resistance in Mycoplasma genitalium

- No Such Thing as Chronic Q Fever
EMERGING INFECTIOUS DISEASES May 2017
- Population Responses during the Pandemic Phase of the Influenza A(H1N1)pdm09 Epidemic, Hong Kong, China

- Survey of Treponemal Infections in Free-Ranging and Captive Macaques, 1999-2012

- Phenotypic and Genotypic Shifts in Hepatitis B Virus in Treatment-Naive Patients, Taiwan, 2008-2012

- Reassortant Clade 2.3.4.4 Avian Influenza A(H5N6) Virus in a Wild Mandarin Duck, South Korea, 2016

- Amoxicillin and Ceftriaxone as Treatment Alternatives to Penicillin for Maternal Syphilis

- Azithromycin Resistance and Decreased Ceftriaxone Susceptibility in Neisseria gonorrhoeae, Hawaii, USA

- Regional Transmission of Salmonella Paratyphi A, China, 1998-2012

- Exposure Risk for Infection and Lack of Human-to-Human Transmission of Mycobacterium ulcerans Disease, Australia

- Virulence Analysis of Bacillus cereus Isolated after Death of Preterm Neonates, Nice, France

- The Discovery of Penicillin-New Insights after More than 75 years of Clinical Use 